\title{
Examining molecular biology in humans
}

\author{
Alex Parker 1 Ian McCaffery ${ }^{2}$, and Scott D. Patterson ${ }^{2}$ \\ ${ }^{1}$ Molecular Sciences, Amgen Inc., Cambridge, MA, USA and ${ }^{2}$ Molecular Sciences, Amgen, Inc., Thousand Oaks, CA, USA
}

BioTechniques 46:358-360 (April 2009 Special Issue) doi 10.2144/000113141

Keywords: imaging; genomics; molecular biology; disease pathophysiology; technology development

Significant advances in biological knowledge have been made through the application of genomic, proteomic, and metabolomic technologies to the interrogation of static samples in model systems of human disease. The integration of the results of these technologies under the banner of systems biology holds much promise. In this forward-looking essay, we posit that to fully understand human biology, such measurements not only need to be integrated, but conducted in humans in real time through the merging of molecular biology and imaging technologies.

\section{Introduction}

The evolution of our understanding of cell biology has been driven by the steady advance of available technologies for analysis of nucleic acids, proteins, and metabolites, culminating in their more recent implementation as 'omic (or massively parallel) applications. These approaches provide a static view of a biological sample obtained at a discrete point in time, with the temporal dimension being captured through multiple sampling over time. If the entity to be studied is an organism, this often requires removal of the particular tissue(s) under investigation. From such measurements of in vitro systems and in vivo model systems, reconstruction of organismal physiology (or pathophysiology) is attempted.

Here, we will consider how the landscape of biological understanding might change over the next 25 years and what technological advances such change would require. We will examine those technologies that hold the potential to enhance our understanding of human physiology, and therefore provide valuable information for the introduction of more efficacious therapeutics. It should also be noted that this essay is not intended to be an extensive review of the literature of any of the fields mentioned; only a few representative references have been included. We theorize that in coming decades, molecular biology will be interrogated directly in humans through a merging of the technologies of molecular biology and imaging, thereby enabling real-time experimental biology in the appropriate physiological setting using non-invasive and non-destructive techniques.

Approaches that permit continuous, real-time data collection through various tagging methodologies coupled with highresolution imaging (1) remain at an early stage of development and some are only relevant to in vitro experimentation. Development of real-time imaging models for preclinical research is being realized through the use of engineered transgenic animals or xenograft cell lines that express reporter constructs (e.g., luciferase) under control of promoters that confer cell type-specific expression or responsiveness to specific physiological conditions. These approaches have extended as far as micro-positron emission tomography (PET) imaging of gene expression using the HSV thymidine kinase, which can be imaged through the use of $\left[8-^{-18} \mathrm{~F}\right]$-fluoroganciclovir (2), and more recently, the determination of phosphorylation through optical bioluminescence imaging of a genetically encoded complementation system (3). Although useful in preclinical context to monitor real-time changes, these approaches do not appear directly translatable to clinical development. However, given the pace of progress in the PET imaging probe field, it is not difficult to imagine that the ability to image activity of specific endogenous enzymes is not too far in the future. The biggest limitation to these approaches comes in the form of our limited understanding of the basic biology behind most underlying cellular processes in vivo: where they are coupled with the dynamic extracellular physiological environment.

A major goal of biological research is to provide a greater understanding of human physiology as it relates to pathological processes involved in disease. Much of our knowledge of pathogenesis and disease progression originates from studies using animal models of disease, which are often poor reflections of the human counterpart (4). Furthermore, many animal model systems involve the use of inbred animals and thus fail to represent the true heterogeneity of human disease that arises from polymorphisms and mutations.

A more complete understanding of the dynamic processes that underlie human physiology at the level of cellular regulatory processes would ultimately inform targeted and precise pharmacological intervention with the aim of modifying the course or symptoms of disease. Although achievements have been made in the rational design of drugs, these efforts are limited by our lack of understanding of the molecular basis of many diseases and the adaptive nature of human physiology; the result is efficacy that is limited to patient subsets and idiosyncratic toxicology (5). As such, our ability to accurately determine the best targets for therapeutic pharmacologic intervention, as well as which patients will benefit from a particular intervention, is restricted. When insights are gained into these questions, it is often only during later stages of the drug development process.

Our understanding of disease heterogeneity and complex regulatory processes has benefitted tremendously from the field of genomics, which is advancing at a rapid pace. Already, we are beginning to uncover the spectrum of variability in human physiology through association studies that correlate genomic changes with a specific phenotype (6). DNA sequence-based genomics in particular is advancing exponentially; it is not, therefore, unrealistic to consider complete genomic characterization of individuals and even of multiple specific tissues with respect to sequence, methylation, copy number variations $(\mathrm{CNV})$, chromosomal rearrangement, and DNA-protein interactions in the coming years. In the infectious-disease context, deepsequencing methods could be deployed to exhaustively catalog the viral, bacterial, and fungal communities inhabiting various areas of an individual's body (7). Similarly, the analyses of RNA, including splice-variants, and sub-cellular and serum/plasma-based proteomics, are undergoing revolutionary changes that will enable extensive, quantitative characterization of much of the molecular activity within a cell. Although many questions have yet to be studied, what is self-evident from current genomics research is that the information encoded by the genome, transcriptome, and proteome is orders of magnitude more complex than was 
once thought, illuminating how far we are away from completely grasping biological processes.

The first advance that we believe would have a significant impact is the intersection of multiple high-content' omic data sets (systems biology), which will help us understand the physiological relevance of genomic variations (SNPs, CNVs, etc.) as they relate to cellular processes. This, in turn, will allow us to focus on key analytes that need to be monitored in humans prior to therapy, for design of personalized therapies, and during treatment to monitor response. This approach would allow the development of probes, or more likely combinations of probes, that could be imaged in vivo to report on the function of specific cell types that are drivers of disease, thereby eliminating one of the existing limitations in tissue-based analyses, which rely on infrequent biopsies or archival tissue samples. Farther in the future, we envision comprehensive genomic and physiological analyses at the single-cell level. Currently, measurement of all of the aforementioned properties of interest in a single cell lies beyond our grasp. The ability to fully characterize the nucleic acids or other analytes contained in a single cell, rather than having to examine the average of many cells sampled together, would revolu- tionize our understanding of the interrelationships of cells in the context of their tissue [both normal and diseased (e.g., malignant tumor cells)]. Being able to simultaneously interrogate the proteome and phosphoproteome of the cell would allow an unprecedented view of the molecular machinery of life at its most fundamental level, representing a significant breakthrough for both disease research and developmental biology.

Consider a tissue section. At present there are a few methods that enable the measurement of specific analytes while preserving their cellular context. Immunohistochemistry (IHC) has long been able to achieve this aim, and more quantitative approaches are now in routine research use, [for example, laser scanning cytometry (8)]. These technologies, while powerful, have limitations, including sensitivity, restrictions on the range of analytes that can be measured, and limited multiplexing capabilities. Imaging mass spectrometry-although a higher-content platform that has greater spatial resolution-cannot interrogate the entire proteome (9). Other than in situ hybridization or in situ PCR, most genomic analyses require cells to be removed from their tissue context and lysed prior to analysis.
The next level of technological advance we forsee is the capacity to comprehensively query the individual genomic and physiological profiles of a series of cells while they remain in their tissue-level context. This capability would be of particular benefit in the field of oncology. Tumors are known to exhibit varying degrees of heterogeneity among the tumor cells themselves, driven by variegated genomic alterations within the tumor cell population. This phenomenon is believed to drive the course of the disease and the response of the tumor to therapy, including development of acquired resistance. The examination of tumor heterogeneity has to date been limited by the available technologies as described above $(10,11)$. Although studies similar to these cited clearly demonstrate tumor heterogeneity, it will require knowledge of the full complement of genomic aberrations (and the resulting effects on all nucleic acid and protein species) at the level of individual cells within their in vivo tissue context for complete understanding of the disease process and the effective management of therapy. Development of molecular probes that can be multiplexed to a much higher degree than is presently feasible is one way in which this may be accomplished. Development of existing methods of labeling probes

\section{How can you do great research on a tight budget?}

\section{Use BrightLine Basic" "no burn-out" fluorescence filters.}

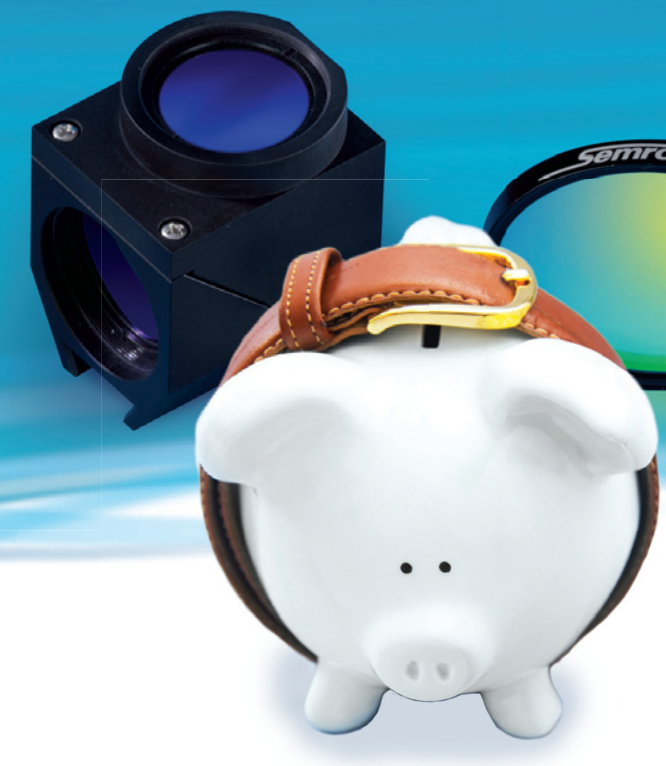

Only Semrock offers you a complete range of

filters from best value to best performance

- all backed by a five-year warranty.

Visit www.semrock.com/freecatalog to request a full-color catalog. 
for spatial readouts could exploit the broad range of emission from quantum dots (12) or other chemistries, such as more sophisticated resonant energy transfer systems, or molecular entities whose reporter function can be toggled on or off by the presence of, for example, magnetic or radio frequency fields. These chemistries would undoubtedly require more precise and sensitive imaging instruments or other approaches yet to be developed. The ability to achieve such goals in the future does not seem out of the realm of possibility.

The advances in molecular biology discussed above would provide an unprecedented understanding of important disease processes at the cellular level. As described, they would require specific probes targeting, for example, genomic alterations or transcripts of key genes, to allow interrogation of the specific cell types that drive a given disease process. In the coming decades, we hope that tools of this kind could then be adapted to enable in vivo interrogation of an even broader range of analytes in humans - rather than in resected tissue-in a relatively non-invasive and non-destructive manner. It is reasonable to expect to gain an increased understanding of disease states and treatment response by examining a range of molecules and molecular processes in human tissues in real time. The ability to interrogate human physiology in vivo is currently rather limited. While the resolution of different imaging techniques is improving and the methods that can be employed in humans are expanding-for example, MRI for soft tissue imaging in tissue remodeling (13) — most available methods query physiological or morphological changes at the level of tissues or organs, which is far different from the scale of individual cells or molecules. Advances have been made in the detection of tumor cells using radiolabeled antibodies that bind specifically to tumor cells [e.g., in the development and monitoring of Rituxan therapy in lymphoma (14)] as well as in the detection of cell proliferation using PET imaging (15). Although the use of tagged antibodies to image tissues is developing quickly, these technologies are non-multiplexable and restricted by the current relatively low resolution of PET imaging technology. Recent advances that may herald a change in this area include optical imaging of antibodybound molecules (16) or specific enzymatic activities (17) in small animals, bringing the level of specificity to single molecular species, if not yet at a molecular scale.

Imagine the power of being able to interrogate cancer patients for a range of specific somatic mutations in real time, while simultaneously tracking the phosphorylation state of key cellular second messengers (ERK, STATs, $\mathrm{AKT}$, etc.) within in situ tumor tissue during the course of therapy. A researcher could answer the question of tumor heterogeneity within an individual, as well as follow what happens in response to therapy over time. Once achieved, this could no doubt be applied to other pathophysiologies. Such tools could potentially also represent superior early detection diagnostics, enabling interventions earlier in the disease processes before symptoms representing pathogenic processes become manifest.

By making it possible to interrogate multiple different analytes simultaneously within humans in real time, the future technologies we envision here could enable the next generation of clinical scientists to build on and synthesize all of the knowledge gained from in vitro and in vivo animal studies to achieve a truly deeper and more integrative understanding of human physiology. Before this novel synthesis can be achieved, however, one additional challenge must be met-the informatic challenge. Although new computational approaches are being developed to store and analyze the massive $n$-dimensional data sets that current technologies routinely generate, the increases in size and complexity of such data will only accelerate as new technologies come online. Seeing is still believing and humans can probably best conceptualize what is going on at the organismal level if the molecules and pathways of interest can be visualized-that is, development of the means to turn massively complex data matrices into representations that can be understood and acted on by humans will be one of the key challenges of the next three decades. Although this was an exercise in what we would like to see in a few decades, the wishes expressed in this article don't seem that far fetched given the pace of molecular analytic technology development.

\section{Acknowledgments}

The authors declare no competing interests.

\section{References}

1. Cohen, A.A., N. Geva-Zatorsky, E. Eden, M. Frenkel-Morgenstern, I. Issaeva, A. Sigal, R. Milo, C. Cohen-Saidon, et al. 2008. Dynamic proteomics of individual cancer cells in response to a drug. Science 322:1511-1516.

2. Gambhir, S.S., J.R. Barrio, L. Wu, M. Iyer, M. Namavari, N. Satyamurthy, E. Bauer, C. Parrish, et al. 1998. Imaging of adenoviral-directed herpes simplex virus type 1 thymidine kinase reporter gene expression in mice with radiolabeled ganciclovir. J. Nucl. Med. 39:2003-2011.

3. Chan, C.T., R. Paulmurugan, R.E. Reeves, D. Solow-Cordero, and S.S. Gambhir. 2008. Molecular imaging of phosphorylation events for drug development. Mol Imaging Biol. [Epub ahead of print, Dec. 2, 2008, doi: 10.1007/s11307008-0187-7].
4. Kelland, L.R. 2004. Of mice and men: values and liabilities of the athymic nude mouse model in anticancer drug development. Eur. J. Cancer 40:827-836.

5. Meletiadis, J., S. Chanock, and T.J. Walsh. 2008. Defining targets for investigating the pharmacogenomics of adverse drug reactions to antifungal agents. Pharmacogenomics 9:561-584.

6. Altshuler, D., M.J. Daly, and E.S. Lander. 2008. Genetic mapping in human disease. Science 322:881-888.

7. Nakamura, S., C.S. Yang, N.Sakon, M. Ueda, T. Tougan, A. Yamashita, N. Goto, K. Takahashi, et al. 2009. Direct metagenomic detection of viral pathogens in nasal and fecal specimens using an unbiased high-throughput sequencing approach. PLoS One 4:e4219.

8. Harnett, M.M. 2007. Laser scanning cytometry: understanding the immune system in situ. Nat. Rev. Immunol. 7:897-904.

9. Hardesty, W.M. and R.M. Caprioli. 2008. In situ molecular imaging of proteins in tissues using mass spectrometry. Anal. Bioanal. Chem. 391:899-903.

10. Baisse, B., H. Bouzourene, E.P. Saraga, F.T. Bosman, and J. Benhattar. 2001. Intratumor genetic heterogeneity in advanced human colorectal adenocarcinoma. Int. J. Cancer 93:346352.

11. Losi, L., B. Baisse, H. Bouzourene, and J. Benhattar. 2005. Evolution of intratumoral genetic heterogeneity during colorectal cancer progression. Carcinogenesis 26:916-922.

12. Yu, W.W., E. Chang, R. Drezek, and V.L. Colvin. 2006. Water-soluble quantum dots for biomedical applications. Biochem. Biophys. Res. Commun. 348:781-786.

13. Xu, H., S.F. Othman, and R.L. Magin. 2008. Monitoring tissue engineering using magnetic resonance imaging. J. Biosci. Bioeng. 106:515527.

14.Stopar, T.G., I. Mlinaric-Rascan, J. Fettich, S. Hojker, and S.J. Mather. 2006. (99m) Tc-rituximab radiolabelled by photo-activation: a new non-Hodgkin's lymphoma imaging agent. Eur. J. Nucl. Med. Mol. Imaging 33:53-59.

15. Salskov, A., V.S. Tammisetti, J. Grierson, and H. Vesselle. 2007. FLT: measuring tumor cell proliferation in vivo with positron emission tomography and 3 '-deoxy-3'-[18F]fluorothymidine. Semin. Nucl. Med. 37:429-439.

16. Chang, S.K., I. Rizvi, N. Solban, and T. Hasan. 2008. In vivo optical molecular imaging of vascular endothelial growth factor for monitoring cancer treatment. Clin. Cancer Res. 14:41464153.

17. Izmailova, E.S., N. Paz, H. Alencar, M. Chun, L. Schopf, M. Hepperle, J.H. Lane, G. Harriman, et al.2007. Use of molecular imaging to quantify response to IKK-2 inhibitor treatment in murine arthritis. Arthritis Rheum. 56:117-128.

Received: 11 February 2009; accepted: 20 Feburary 2009.

Address correspondence to Scott Patterson, Molecular Sciences, Amgen, Inc., One Amgen Center Drive, MS 38-3-A, Thousand Oaks, CA 91320, USA.email: spatters@amgen.com 